\title{
On a system of fractional finite difference inclusions
}

\author{
Vahid Ghorbanian and Shahram Rezapour*
}

"Correspondence:

math2010math@gmail.com

Department of Mathematics,

Azarbaijan Shahid Madani

University, Tabriz, Iran

Full list of author information is

available at the end of the article (c) The Author(s) 2017. This article is distributed under the terms of the Creative Commons Attribution 4.0 International License (http://creativecommons.org/licenses/by/4.0/), which permits unrestricted use, distribution, and reproduction in any medium, provided you give appropriate credit to the original author(s) and the source, provide a link to the Creative Commons license, and indicate if changes were made.

\begin{abstract}
By making a special product Banach space and using the famous result of Covitz and Nadler on fixed point of multifunctions we investigate the existence of a solution for a system of fractional finite difference inclusions via some boundary conditions. We provide an example to illustrate our main result.
\end{abstract}

MSC: $34 \mathrm{~A} 60 ; 65 \mathrm{~N} 06$

Keywords: fractional finite difference; Green function; system of inclusions

\section{Introduction}

There are many works about different applied models by using distinct types of fractional derivatives via or without singular kernel ([1-6]), discrete fractional boundary value problems within the Riesz space cases $([7,8])$, finite difference calculations ([9-13]), distinct types of fractional finite difference equations ([14-26]), and some equations including the nabla operator ([27-30]). In fact, working on discrete fractional boundary value problems is useful for modeling in distinct thermal or physical sciences, including steady heat flows, heat-transfer problems, description of anomalous diffusions, and so on. This leads us to working on discrete calculations, whereas there is also rich work on continuous fractional ones. It is well known that each differential equation is a particular case of a related differential inclusion. For this reason, we better investigate fractional inclusions. It seems that researchers of thermal sciences (and some other related fields) will investigate more systems of discrete fractional boundary value inclusions in the future. Recently, some results on fractional finite difference inclusions have been obtained $([18,19])$.

As is well known, the gamma function has some known properties such as $\Gamma(z+1)=$ $z \Gamma(z)$ and $\Gamma(n)=(n-1)$ ! for all $n \in \mathbb{N}$. It is well known that the falling function is defined by $t^{\underline{v}}=\frac{\Gamma(t+1)}{\Gamma(t+1-v)}$ for all $t, v \in \mathbb{R}$ whenever the right-hand side is defined ([31]). If $t+1-v$ is a pole of the gamma function and $t+1$ is not a pole, then we define $t \underline{v}=0([31])$. We can verify that $\nu^{\underline{v}}=\nu \underline{v-1}=\Gamma(\nu+1)$ and $t^{\underline{\nu+1}}=(t-v) t^{\underline{v}}$. We use the notations $\mathbb{N}_{a}=\{a, a+1, a+2, \ldots\}$ for all $a \in \mathbb{R}$ and $\mathbb{N}_{a}^{b}=\{a, a+1, a+2, \ldots, b\}$ for all real numbers $a$ and $b$ whenever $b-a$ is a natural number ([31]). Let $v>0$ be such that $m-1<v \leq m$ for some natural number $m$. Then the $v$ th fractional sum of $f$ based at $a$ is defined by $\Delta_{a}^{-v} f(t)=\frac{1}{\Gamma(v)} \sum_{s=a}^{t-v}(t-\sigma(s)) \frac{v-1}{} f(s)$ for all $t \in \mathbb{N}_{a+v}$ ([12]). We consider the trivial case $\Delta_{a}^{-0} f(t)=f(t)$ for $t \in \mathbb{N}_{a}$. Similarly, we define $\Delta_{a}^{v} f(t)=\frac{1}{\Gamma(-v)} \sum_{k=a}^{t+v}(t-\sigma(k)) \frac{-v-1}{-} f(k)$ for all $t \in \mathbb{N}_{a+m-v}$ (see [28] and [32]). 
To use the Covitz-Nadler theorem in our main result, we need to introduce some notion about multifunctions on metric spaces. Let $(X, d)$ be a metric space. Denote by $P(X)$, $2^{X}, P_{c}(X)$, and $P_{c p}(X)$ the class of all subsets, the class of all nonempty subsets, the class of all closed subsets, and the class of all compact subsets of $X$, respectively. A mapping $Q: X \rightarrow 2^{X}$ is called a multifunction on $X$, and $u \in X$ is called a fixed point of $Q$ whenever $u \in Q u$. The (generalized) Pompeiu-Hausdorff metric $H_{d}$ on $P_{c}(X)$ is defined as $H_{d}(A, B)=\max \left\{\sup _{a \in A} d(a, B), \sup _{b \in B} d(A, b)\right\}$, where $d(A, b)=\inf _{a \in A} d(a, b)$ (see [33] and [34]). A multifunction $T: X \rightarrow 2^{X}$ is called a contraction if there exists $\lambda \in(0,1)$ such that $H_{d}(T(x), T(y)) \leq \lambda d(x, y)$ for all $x, y \in X$. In 1970, Covitz and Nadler [35] proved that each contractive closed-valued multifunction on a complete metric space has a fixed point.

In 2011, Goodrich [36] investigated the general discrete fractional boundary problem

$$
\left\{\begin{array}{l}
-\Delta^{v} y(t)=f(t+v-1, y(t+v-1)) \\
\alpha y(v-2)-\beta \Delta y(v-2)=0 \\
\gamma y(v+b)-\delta \Delta y(v+b)=0
\end{array}\right.
$$

where $t \in[0, b]_{\mathbb{N}_{0}}, v \in(1,2]$ and $\alpha \gamma+\alpha \delta+\beta \gamma \neq 0$ with $\alpha, \beta, \gamma, \delta \geq 0$. In 2015, by using idea of [36], Baleanu, Rezapour, and Salehi [18] investigated the existence of a solution for the fractional finite difference inclusion

$$
\left\{\begin{array}{l}
\Delta^{v} x(t) \in F\left(t, x(t), \Delta x(t), \Delta^{2} x(t)\right) \\
\xi x(v-3)+\beta \Delta x(v-3)=0 \\
x(\eta)=0 \\
\gamma x(b+v)+\delta \Delta x(b+v)=0
\end{array}\right.
$$

where $\eta \in \mathbb{N}_{v-2}^{b+v-1}, 2<v<3$, and $F: \mathbb{N}_{v-3}^{b+v+1} \times \mathbb{R} \times \mathbb{R} \times \mathbb{R} \rightarrow 2^{\mathbb{R}}$ is a compact-valued multifunction. Also, they investigated the fractional finite difference inclusion $\Delta_{\mu-2}^{\mu} x(t) \in$ $F(t, x(t), \Delta x(t))$ via the boundary conditions $\Delta x(b+\mu)=A$ and $x(\mu-2)=B$, where $1<\mu \leq 2, A, B \in \mathbb{R}$, and $F: \mathbb{N}_{\mu-2}^{b+\mu+2} \times \mathbb{R} \times \mathbb{R} \rightarrow 2^{\mathbb{R}}$ is a compact-valued multifunction in 2016 ([19]). By mixing ideas of the works, we investigate the existence of a solution for the $k$-dimensional system of fractional difference inclusions

$$
\left\{\begin{aligned}
\Delta_{v_{1}-1}^{v_{1}} x_{1}(t) \in & F_{1}\left(t, x_{1}(t), \ldots, x_{k}(t), \Delta x_{1}(t), \ldots, \Delta x_{k}(t), \Delta^{2} x_{1}(t), \ldots, \Delta^{2} x_{k}(t),\right. \\
& \left.\Delta_{v_{1}+1}^{\mu_{11} x_{1}}(t), \ldots, \Delta_{v_{1}+1}^{\mu_{1 k}} x_{k}(t), \Delta_{v_{1}+1}^{\gamma_{11}} x_{1}(t), \ldots, \Delta_{v_{1}+1}^{\gamma_{1 k}} x_{k}(t)\right), \\
\Delta_{v_{2}-1}^{v_{2}} x_{2}(t) \in & F_{2}\left(t, x_{1}(t), \ldots, x_{k}(t), \Delta x_{1}(t), \ldots, \Delta x_{k}(t), \Delta^{2} x_{1}(t), \ldots, \Delta^{2} x_{k}(t),\right. \\
& \left.\Delta_{v_{2}+1}^{\mu_{21}} x_{1}(t), \ldots, \Delta_{v_{2}+1}^{\mu_{2 k}} x_{k}(t), \Delta_{v_{2}+1}^{\gamma_{21}} x_{1}(t), \ldots, \Delta_{v_{2}+1}^{\gamma_{2 k}} x_{k}(t)\right), \\
& \\
\Delta_{v_{k}-1}^{v_{k}} x_{k}(t) \in & F_{k}\left(t, x_{1}(t), \ldots, x_{k}(t), \Delta x_{1}(t), \ldots, \Delta x_{k}(t), \Delta^{2} x_{1}(t), \ldots, \Delta^{2} x_{k}(t),\right. \\
& \left.\Delta_{v_{k}+1}^{\mu_{k 1}} x_{1}(t), \ldots, \Delta_{v_{k}+1}^{\mu_{k}} x_{k}(t), \Delta_{v_{k}+1}^{\gamma_{k 1}} x_{1}(t), \ldots, \Delta_{v_{k}+1}^{\gamma_{k k}} x_{k}(t)\right),
\end{aligned}\right.
$$

with boundary conditions $x_{i}\left(v_{i}-1\right)=x_{i}\left(v_{i}\right)=x_{i}\left(b+v_{i}\right)=0$, where $x_{i}: \mathbb{N}_{v_{i}-1}^{b+v_{i}} \rightarrow \mathbb{R}, b \in \mathbb{N}_{0}, 0<$ $\mu_{i j}<1,1<\gamma_{i j}<2,2<v_{i}<3$, and $F_{i}: \mathbb{N}_{v_{i}-1}^{b+v_{i}} \times \mathbb{R}^{5 k} \rightarrow 2^{\mathbb{R}}$ are compact-valued multifunction for $i=1,2, \ldots, k$ and $j=1,2, \ldots, k$.

For prove of our main results, we need the following results. 
Lemma 1.1 ([11]) Let $a \in \mathbb{R}, v>0$ with $m-1<v \leq m$, and $\mu>0$. Then, $\Delta(t-a) \underline{\mu}=\mu(t-$ a) $\stackrel{\mu-1}{\underline{1}}$ for all $t$ for which both sides are well defined. Also, $\Delta_{a+\mu}^{-v}(t-a) \underline{\mu}=\mu-\underline{-v}(t-a) \stackrel{\mu+v}{\text { for }}$ $t \in \mathbb{N}_{a+\mu+\nu}$ and $\Delta_{a+\mu}^{v}(t-a)^{\underline{\mu}}=\mu \underline{\underline{v}}(t-a) \stackrel{\mu-v}{\underline{v}}$ for $t \in \mathbb{N}_{a+\mu+m-\nu}$.

Lemma 1.2 ([26]) Let $\mu>0$ with $m-1<\mu \leq m, a \in \mathbb{R}$, and $f: \mathbb{N}_{a} \rightarrow \mathbb{R}$ be a map. Then, $\Delta_{a+m-\mu}^{-\mu} \Delta_{a}^{\mu} f(t)=f(t)+c_{1}(t-a-m+\mu) \frac{\mu-1}{2}+c_{2}(t-a-m+\mu) \frac{\mu-2}{2}+\cdots+c_{m}(t-a-m+\mu) \frac{\mu-m}{n}$, where $c_{1}, \ldots, c_{m} \in \mathbb{R}$ are some constants.

\section{Main results}

Now, we are ready to provide our main results.

Lemma 2.1 Let $2<\mu \leq 3$, and $y: \mathbb{N}_{2}^{b} \rightarrow \mathbb{R}$ be a map. Then $x_{0}$ is a solution for the fractional finite difference equation

$$
\Delta_{\mu-1}^{\mu} x(t)=y(t)
$$

via the boundary conditions $x(\mu-1)=x(\mu)=x(b+\mu)=0$ if and only if $x_{0}$ is a solution for the fractional sum equation $x(t)=\sum_{s=2}^{b} G(t, s) y(s)$, where

$$
G(t, s)=\frac{(t-\sigma(s))^{\mu-1}}{\Gamma(\mu)}-\frac{(t-2)^{\underline{\mu-1}}(b+\mu-\sigma(s)) \frac{\mu-1}{}}{\Gamma(\mu)(b+\mu-2)^{\mu-1}}
$$

for $s \leq t-\mu$ and $G(t, s)=\frac{-(t-2) \frac{\mu-1}{\left(b+\mu-\sigma(s) \frac{\mu-1}{~}\right.}}{\Gamma(\mu)(b+\mu-2)^{\frac{\mu-1}{2}}}$ for $t-\mu+1 \leq s$.

Proof Suppose that $x_{0}$ satisfies equation (2) with $x_{0}(\mu-1)=x_{0}(\mu)=x_{0}(b+\mu)=0$. Then, $\Delta_{\mu-1}^{\mu} x_{0}(t)=y(t)$ for $t \in \mathbb{N}_{2}^{b}$ and $\Delta_{2}^{-\mu} \Delta_{\mu-1}^{\mu} x(t)=\Delta_{2}^{-\mu} y(t)$ for $t \in \mathbb{N}_{\mu-1}^{b+\mu}$. Now, using Lemma 1.2, we get $x_{0}(t)=c_{1}(t-2) \frac{\mu-1}{\underline{c}}+c_{2}(t-2) \stackrel{\mu-2}{\underline{\mu}}+c_{3}(t-2) \frac{\mu-3}{+}+\frac{1}{\Gamma(\mu)} \sum_{s=2}^{t-\mu}(t-\sigma(s))^{\mu-1} y(s)$ for $t \in \mathbb{N}_{\mu-1}^{b+\mu}$. Using the boundary condition $x(\mu-1)=0$, we obtain

$$
0=c_{1}(\mu-3) \frac{\mu-1}{2}+c_{2}(\mu-3) \frac{\mu-2}{2}+c_{3}(\mu-3) \frac{\mu-3}{\Gamma}+\frac{1}{\Gamma(\mu)} \sum_{s=2}^{-1}(\mu-1-\sigma(s)) \frac{\mu-1}{=} y(s) .
$$

Since $(\mu-3) \frac{\mu-1}{\mu}=(\mu-3) \stackrel{\mu-2}{=}=0$ and $\frac{1}{\Gamma(\mu)} \sum_{s=2}^{-1}(\mu-1-\sigma(s)) \stackrel{\mu-1}{\underline{y}} y(s)=0$, we get $c_{3}=0$. Since $x(\mu)=0,0=c_{1}(\mu-2) \frac{\mu-1}{n}+c_{2}(\mu-2) \frac{\mu-2}{2}+\frac{1}{\Gamma(\mu)} \sum_{s=2}^{0}(\mu-\sigma(s)) \frac{\mu-1}{y} y(s)$. Since $(\mu-2) \underline{\mu-1}=0$ and $\frac{1}{\Gamma(\mu)} \sum_{s=2}^{0}(\mu-\sigma(s)) \stackrel{\mu-1}{y} y(s)=0$, we get $c_{2}=0$. Since $x(b+\mu)=0$, we get

$$
0=c_{1}(b+\mu-2) \frac{\mu-1}{\Gamma}+\frac{1}{\Gamma(\mu)} \sum_{s=2}^{b}(b+\mu-\sigma(s))^{\frac{\mu-1}{-}} y(s),
$$

and so $c_{1}=-\frac{1}{\Gamma(\mu)(b+\mu-2) \underline{\mu-1}} \sum_{s=2}^{b}(b+\mu-\sigma(s)) \frac{\mu-1}{y} y(s)$. Hence,

$$
\begin{aligned}
& x_{0}(t)=\frac{-(t-2) \underline{\mu-1}}{\Gamma(\mu)(b+\mu-2)^{\mu-1}} \sum_{s=2}^{b}(b+\mu-\sigma(s))^{\frac{\mu-1}{y}} y(s)+\frac{1}{\Gamma(\mu)} \sum_{s=2}^{t-\mu}(t-\sigma(s))^{\frac{\mu-1}{}} y(s) \\
& =\sum_{s=2}^{b} G(t, s) y(s) \text {. }
\end{aligned}
$$


Now, let $x_{0}$ be a solution for the sum equation $x(t)=\sum_{s=2}^{b} G(t, s) y(s)$. Then,

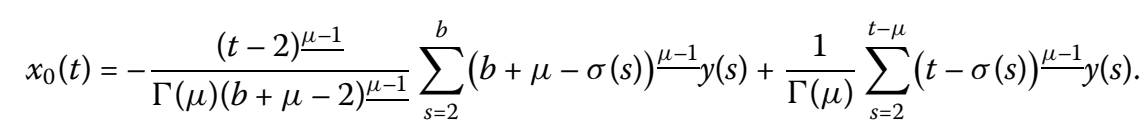

Since $\frac{1}{\Gamma(\mu)} \sum_{s=2}^{-1}(\mu-1-\sigma(s)) \frac{\mu-1}{2} y(s)=\frac{1}{\Gamma(\mu)} \sum_{s=2}^{0}(\mu-\sigma(s)) \frac{\mu-1}{\mu} y(s)=0$ and $(\mu-3) \underline{\mu-1}=(\mu-$ 3) $\frac{\mu-2}{2}=(\mu-2) \frac{\mu-1}{2}=0$, a simple calculation shows that $x(\mu-1)=x(\mu)=x(b+\mu)=0$. On the other hand, it is easy to check that

$$
x_{0}(t)=c_{1}(t-2) \frac{\mu-1}{2}+c_{2}(t-2) \frac{\mu-2}{2}+c_{3}(t-2) \frac{\mu-3}{\Gamma}+\frac{1}{\Gamma(\mu)} \sum_{s=2}^{t-\mu}(t-\sigma(s))^{\frac{\mu-1}{y}} y(s)
$$

is a solution for the equation $\Delta_{\mu-1}^{\mu} x(t)=y(t)$.

Now, we are ready to provide our result on the existence of a solution for the $k$ dimensional system of fractional finite difference inclusions (1). Let $i \in\{1, \ldots, k\}$ be given, and $\mathcal{X}_{i}$ be the set of all functions $x: \mathbb{N}_{v_{i}-1}^{b+v_{i}} \rightarrow \mathbb{R}$ endowed with the norm

$$
\begin{aligned}
\|x\|_{i}= & \max _{\substack{t \in \mathbb{N}_{v_{i}-1}^{b+v_{i}}\\
}}|x(t)|+\max _{t \in \mathbb{N}_{v_{i}-1}^{b+v_{i}}}|\Delta x(t)|+\max _{t \in \mathbb{N}_{v_{i}-1}^{b+v_{i}}}\left|\Delta^{2} x(t)\right| \\
& +\max _{t \in \mathbb{N}_{v_{i}-1}^{b+v_{i}}}\left|\Delta_{v_{i}+1}^{\mu_{i i}} x(t)\right|+\max _{t \in \mathbb{N}_{v_{i}-1}^{b+v_{i}}}\left|\Delta_{v_{i}+1}^{\gamma_{i i}} x(t)\right| .
\end{aligned}
$$

Consider the space $\mathcal{X}=\mathcal{X}_{1} \times \mathcal{X}_{2} \times \cdots \times \mathcal{X}_{k}$ with the norm $\left\|\left(x_{1}, x_{2}, \ldots, x_{k}\right)\right\|_{\mathcal{X}}=\sum_{i=1}^{k}\left\|x_{i}\right\|_{i}$. We show that $\left(\mathcal{X},\|\cdot\|_{\mathcal{X}}\right)$ is a Banach space. Let $\left\{x_{n}\right\}$ be a Cauchy sequence in $\mathcal{X}$, and let $\epsilon>$ 0 be arbitrary. Choose a natural number $N$ such that $\left\|x_{n}-x_{m}\right\|<\epsilon$ for all $m, n>N$. Then we get $\max _{t \in \mathbb{N}_{v}^{b+v+2}}\left|x_{n}(t)-x_{m}(t)\right|<\epsilon, \max _{t \in \mathbb{N}_{v}^{b+v+2}}\left|\Delta x_{n}(t)-\Delta x_{m}(t)\right|<\epsilon, \max _{t \in \mathbb{N}_{v}^{b+v+2}} \mid \Delta^{2} x_{n}(t)-$ $\Delta^{2} x_{m}(t)\left|<\epsilon, \max _{t \in \mathbb{N}_{v}^{b+v+2}}\right| \Delta^{\mu} x_{n}(t)-\Delta^{\mu} x_{m}(t) \mid<\epsilon$, and $\max _{t \in \mathbb{N}_{v}^{b+v+2}}\left|\Delta^{\gamma} x_{n}(t)-\Delta^{\gamma} x_{m}(t)\right|<\epsilon$. Since $\mathbb{R}$ is complete, there are real numbers $x(t), z(t), w(t), p(t)$, and $q(t)$ such that $x_{n}(t) \rightarrow$ $x(t), \Delta x_{n}(t) \rightarrow z(t), \Delta^{2} x_{n}(t) \rightarrow w(t), \Delta^{\mu} x_{n}(t) \rightarrow p(t)$, and $\Delta^{\gamma} x_{n}(t) \rightarrow q(t)$ for all $t \in \mathbb{N}_{v}^{b+v+2}$. Note that $\Delta x_{n}(t)=x_{n}(t+1)-x_{n}(t)$, and so $\Delta x(t)=x(t+1)-x(t)=z(t)$. Similarly, we get $\Delta^{2} x(t)=w(t)$. Also, we have $\Delta^{\mu} x_{n}(t)=\frac{1}{\Gamma(-\mu)} \sum_{s=0}^{t+\mu}(t-\sigma(s)) \stackrel{-\mu-1}{x_{n}}(s)$. Since $x_{n}(s) \rightarrow x(s)$, we get $\Delta^{\mu} x(t)=p(t)$. Similarly, we have $\Delta^{\gamma} x(t)=q(t)$. This implies that there exists a natural number $M$ such that $\left|x_{n}(t)-x(t)\right|<\frac{\epsilon}{5},\left|\Delta x_{n}(t)-\Delta x(t)\right|<\frac{\epsilon}{5},\left|\Delta^{2} x_{n}(t)-\Delta^{2} x(t)\right|<\frac{\epsilon}{5}$, $\left|\Delta^{\mu} x_{n}(t)-\Delta^{\mu} x(t)\right|<\frac{\epsilon}{5}$, and $\left|\Delta^{\gamma} x_{n}(t)-\Delta^{\gamma} x(t)\right|<\frac{\epsilon}{5}$ for all $t \in \mathbb{N}_{v}^{b+v+2}$ and $n>M$. Thus,

$$
\begin{aligned}
\left\|x_{n}-x\right\|= & \max _{t \in \mathbb{N}_{\nu}^{b+v+2}}\left|x_{n}(t)-x(t)\right|+\max _{t \in \mathbb{N}_{\nu}^{b+v+2}}\left|\Delta x_{n}(t)-\Delta x(t)\right|+\max _{t \in \mathbb{N}_{v}^{b+v+2}}\left|\Delta^{2} x_{n}(t)-\Delta^{2} x(t)\right| \\
& +\max _{t \in \mathbb{N}_{v}^{b+v+2}}\left|\Delta^{\mu} x_{n}(t)-\Delta^{\mu} x(t)\right|+\max _{t \in \mathbb{N}_{v}^{b+v+2}}\left|\Delta^{\gamma} x_{n}(t)-\Delta^{\gamma} x(t)\right|<\epsilon
\end{aligned}
$$

for all $n>M$. This shows that $\mathcal{X}$ is a Banach space. Define the set of selections of $F_{i}$ at $\left(x_{1}, \ldots, x_{k}\right) \in \mathcal{X}$ by

$$
\begin{aligned}
S_{F_{i},\left(x_{1}, x_{2}, \ldots, x_{k}\right)=} & \left\{y: \mathbb{N}_{2}^{b} \rightarrow \mathbb{R}: y(t) \in F_{i}\left(t, x_{1}(t), \ldots, x_{k}(t), \Delta x_{1}(t), \ldots, \Delta x_{k}(t),\right.\right. \\
& \Delta^{2} x_{1}(t), \ldots, \Delta^{2} x_{k}(t), \Delta_{v_{i}+1}^{\mu_{i 1}} x_{1}(t), \ldots, \Delta_{v_{i}+1}^{\mu_{i k}} x_{k}(t), \\
& \left.\left.\Delta_{v_{i}+1}^{\gamma_{i 1}} x_{1}(t), \ldots, \Delta_{v_{i}+1}^{\gamma_{i k}} x_{k}(t)\right) \text { for all } t \in \mathbb{N}_{2}^{b}\right\}
\end{aligned}
$$


for $x=\left(x_{1}, \ldots, x_{k}\right) \in \mathcal{X}$ and $i=1, \ldots, k$. We say that a function $\left(x_{1}, x_{2}, \ldots, x_{k}\right) \in \mathcal{X}$ is a solution for the $k$-dimensional system of inclusions if there exist real-valued functions $y_{1}, \ldots, y_{k}$ on $\mathbb{N}_{2}^{b}$ such that

$$
\begin{gathered}
y_{i}(t) \in F_{i}\left(t, x_{1}(t), \ldots, x_{k}(t), \Delta x_{1}(t), \ldots, \Delta x_{k}(t), \Delta^{2} x_{1}(t), \ldots, \Delta^{2} x_{k}(t),\right. \\
\left.\Delta_{v_{i}+1}^{\mu_{i 1}} x_{1}(t), \ldots, \Delta_{v_{i}+1}^{\mu_{i k}} x_{k}(t), \Delta_{v_{i}+1}^{\gamma_{i 1}} x_{1}(t), \ldots, \Delta_{v_{i}+1}^{\gamma_{i k}} x_{k}(t)\right)
\end{gathered}
$$

for all $t \in \mathbb{N}_{2}^{b}, x_{i}(t)=\frac{-(t-2) \stackrel{v_{i}-1}{\underline{v^{\prime}}}}{\Gamma\left(v_{i}\right)\left(b+v_{i}-2\right) \underline{v_{i}-1}} \sum_{s=2}^{b}\left(b+v_{i}-\sigma(s)\right) \frac{v_{i}-1}{y_{i}} y_{i}(s)+\frac{1}{\Gamma\left(v_{i}\right)} \sum_{s=2}^{t-v_{i}}(t-\sigma(s)) \frac{v_{i}-1}{h} y_{i}(s)$ and $x_{i}\left(v_{i}-1\right)=x_{i}\left(v_{i}\right)=x_{i}\left(b+v_{i}\right)=0$ for $i=1, \ldots, k$. Since $F_{i}\left(t, x_{1}(t), \ldots, x_{k}(t)\right) \neq \varnothing$ for $i=$ $1, \ldots, k$, the selection principle implies that $S_{F_{i},\left(x_{1}, x_{2}, \ldots, x_{k}\right)}$ is nonempty.

Theorem 2.2 Suppose that $\psi_{1}, \ldots, \psi_{k}: \mathbb{N}_{v_{i}-1}^{b+v_{i}} \rightarrow \mathbb{R}$ be some maps with

$$
0<L=\sum_{i=1}^{k}\left(\max _{t \in \mathbb{N}_{v_{i}-1}^{b+v_{i}}}\left|\psi_{i}(t)\right|\right)\left(\Lambda_{1}^{i}+\cdots+\Lambda_{5}^{i}\right)<1
$$

where

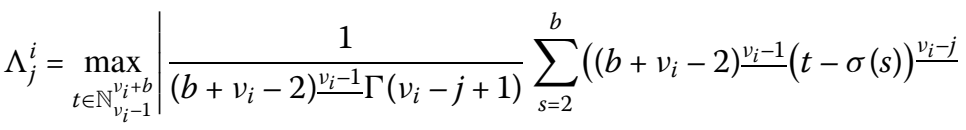

$$
\begin{aligned}
& -(t-2) \frac{v_{i}-j}{\left.\left(b+v_{i}-\sigma(s)\right) \frac{v_{i}-1}{}\right)}
\end{aligned}
$$

for $j=1,2,3$,

$$
\begin{aligned}
& \Lambda_{4}^{i}=\max _{t \in \mathbb{N}_{v_{i}-1}^{v_{i}+b}} \mid \frac{1}{\Gamma\left(v_{i}-\mu_{i i}\right)\left(b+v_{i}-2\right) \frac{v_{i}-1}{m}} \sum_{s=2}^{b}\left(\left(b+v_{i}-2\right) \frac{v_{i}-1}{(t-\sigma(s)) \frac{v_{i}-\mu_{i i}}{}}\right. \\
& -(t-2) \frac{v_{i}-\mu_{i i}}{\left.\left(b+v_{i}-\sigma(s)\right) \frac{v_{i}-1}{}\right) \mid},
\end{aligned}
$$

and

$$
\begin{aligned}
\Lambda_{5}^{i}= & \max _{\substack{t \in \mathbb{N}_{v_{i}-1}^{v_{i}+b}\\
}} \mid \frac{1}{\Gamma\left(v_{i}-\gamma_{i i}\right)\left(b+v_{i}-2\right) \frac{v_{i}-1}{s=2}} \sum_{s=2}^{b}\left(\left(b+v_{i}-2\right) \frac{v_{i}-1}{(t-\sigma(s)) \frac{v_{i}-\gamma_{i i}}{}}\right. \\
& -(t-2) \frac{v_{i}-\gamma_{i i}}{\left.\left(b+v_{i}-\sigma(s)\right) \frac{v_{i}-1}{n}\right) \mid .}
\end{aligned}
$$

Assume that $F_{i}: \mathbb{N}_{v_{i}-1}^{b+v_{i}} \times \mathbb{R}^{5 k} \rightarrow P_{c p}(\mathbb{R})$ is a multifunction such that

$$
H_{d}\left(F_{i}\left(t, x_{1}, \ldots, x_{5 k}\right), F_{i}\left(t, z_{1}, \ldots, z_{5 k}\right)\right) \leq \psi_{i}(t)\left(\sum_{i=1}^{5 k}\left|x_{i}-z_{i}\right|\right)
$$

for $t \in \mathbb{N}_{v_{i}-1}^{b+v_{i}}, i=1, \ldots, k$, and $x_{1}, \ldots, x_{5 k}, z_{1}, \ldots, z_{5 k} \in \mathbb{R}$. Then the system of fractional difference inclusions (1) has a solution. 
Proof Choose $y_{i} \in S_{F_{i},\left(x_{1}, x_{2}, \ldots, x_{k}\right)}$ for $i=1, \ldots, k$. Define

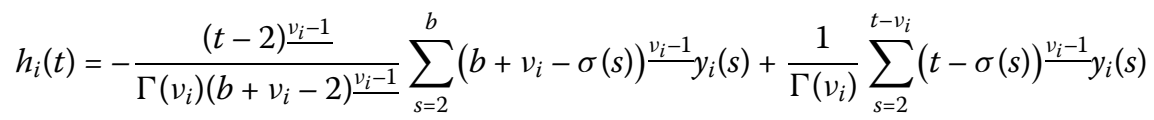

for all $t \in \mathbb{N}_{v_{i}-1}^{b+v_{i}}$. Then $h_{i} \in \mathcal{X}_{i}$, and so the set

$$
\begin{aligned}
\left\{h_{i} \in \mathcal{X}_{i}\right. & \text { there exists } y \in S_{F_{i},\left(x_{1}, x_{2}, \ldots, x_{k}\right)} \text { such that } \\
h_{i}(t)= & -\frac{(t-2) \frac{v_{i}-1}{\Gamma\left(v_{i}\right)\left(b+v_{i}-2\right)}}{{ }^{v_{i}-1}} \sum_{s=2}^{b}\left(b+v_{i}-\sigma(s)\right) \frac{v_{i}-1}{v_{i}} y_{i}(s) \\
& \left.+\frac{1}{\Gamma\left(v_{i}\right)} \sum_{s=2}^{t-v_{i}}(t-\sigma(s))^{\frac{v_{i}-1}{}} y_{i}(s) \text { for all } t \in \mathbb{N}_{v_{i}-1}^{b+\nu_{i}}\right\}
\end{aligned}
$$

is nonempty. Define the operator $T: \mathcal{X} \rightarrow 2^{\mathcal{X}}$ by

$$
T\left(x_{1}, x_{2}, \ldots, x_{k}\right)\left(t_{1}, t_{2}, \ldots, t_{k}\right)=\left(\begin{array}{c}
T_{1}\left(x_{1}, x_{2}, \ldots, x_{k}\right)\left(t_{1}\right) \\
T_{2}\left(x_{1}, x_{2}, \ldots, x_{k}\right)\left(t_{2}\right) \\
\vdots \\
T_{k}\left(x_{1}, x_{2}, \ldots, x_{k}\right)\left(t_{k}\right)
\end{array}\right),
$$

where

$$
\begin{aligned}
& T_{i}\left(x_{1}, x_{2}, \ldots, x_{k}\right)=\left\{f \in \mathcal{X}_{i}: \text { there exists } y_{i} \in S_{F_{i,\left(x_{1}, \ldots, x_{k}\right)}}\right. \text { such that }
\end{aligned}
$$

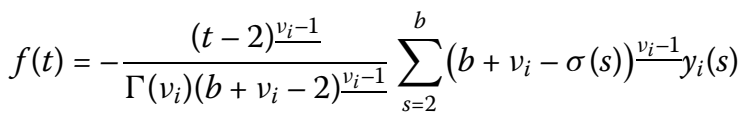

$$
\begin{aligned}
& \left.+\frac{1}{\Gamma\left(v_{i}\right)} \sum_{s=2}^{t-v_{i}}(t-\sigma(s))^{\frac{v_{i}-1}{}} y_{i}(s), \text { for all } t \in \mathbb{N}_{v_{i}-1}^{b+v_{i}}\right\} \text {. }
\end{aligned}
$$

We show that the multifunction $T$ has a fixed point. First, we prove that $T\left(x_{1}, x_{2}, \ldots, x_{k}\right)$ is a closed subset of $\mathcal{X}$ for all $\left(x_{1}, x_{2}, \ldots, x_{k}\right) \in \mathcal{X}$. Let $\left(x_{1}, x_{2}, \ldots, x_{k}\right) \in \mathcal{X}$, and let $\left\{\left(x_{1}^{n}, \ldots, x_{k}^{n}\right)\right\}_{n \geq 1}$ be a sequence in $T\left(x_{1}, x_{2}, \ldots, x_{k}\right)$ with $\left(x_{1}^{n}, \ldots, x_{k}^{n}\right) \rightarrow\left(x_{1}^{0}, \ldots, x_{k}^{0}\right)$. For each $n$, choose $\left(y_{1}^{n}, \ldots, y_{k}^{n}\right) \in S_{F_{1},\left(x_{1}, \ldots, x_{k}\right)} \times S_{F_{2},\left(x_{1}, \ldots, x_{k}\right)} \times \cdots \times S_{F_{k},\left(x_{1}, \ldots, x_{k}\right)}$ such that

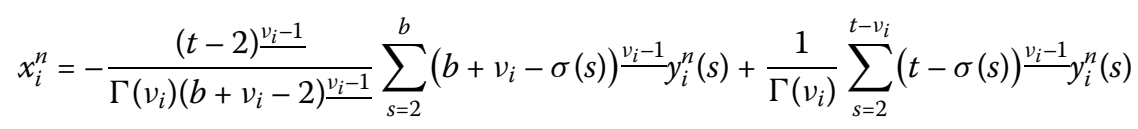

for $t \in \mathbb{N}_{v_{i}-1}^{b+v_{i}}, n \geq 1$ and $i=1, \ldots, k$. Since the multifunctions $F_{1}, \ldots, F_{k}$ are compact-valued, $\left\{y_{i}^{n}\right\}_{n \geq 1}$ has a subsequence that converges to some $y_{i}^{0}: \mathbb{N}_{2}^{b} \rightarrow \mathbb{R}$. We denote this subsequence again by $\left\{y_{i}^{n}\right\}_{n \geq 1}$. It is easy to check that $y_{i}^{0} \in S_{F_{i},\left(x_{1}, \ldots, x_{k}\right)}$ and

$$
\begin{aligned}
& x_{i}^{n}(t) \rightarrow x_{i}^{0}(t)=-\frac{(t-2)^{v_{i}-1}}{\Gamma\left(v_{i}\right)\left(b+v_{i}-2\right) \frac{v_{i}-1}{\underline{n}}} \sum_{s=2}^{b}\left(b+v_{i}-\sigma(s)\right)^{\frac{v_{i}-1}{}} y_{i}^{0}(s) \\
& +\frac{1}{\Gamma\left(v_{i}\right)} \sum_{s=2}^{t-v_{i}}(t-\sigma(s)) \frac{v_{i}-1}{y_{i}^{0}(s)}
\end{aligned}
$$


for all $t \in \mathbb{N}_{v_{i}-1}^{b+v_{i}}$ and $i=1, \ldots, k$. This implies that $x_{i}^{0} \in T_{i}\left(x_{1}, \ldots, x_{s}\right)$ for all $i=1, \ldots, k$. Hence, $\left(x_{1}^{0}, \ldots, x_{k}^{0}\right) \in T\left(x_{1}, \ldots, x_{k}\right)$, and so the multifunction $T$ has closed values. Since $T$ is a compact-valued multifunction, it is easy to see that $T\left(x_{1}, \ldots, x_{k}\right)$ is a bounded set in $\mathcal{X}$ for all $\left(x_{1}, \ldots, x_{k}\right) \in \mathcal{X}$. Let $\left(u_{1}, \ldots, u_{k}\right),\left(v_{1}, \ldots, v_{k}\right) \in \mathcal{X},\left(h_{1}, \ldots, h_{k}\right) \in T\left(u_{1}, \ldots, u_{k}\right)$ and $\left(h_{1}^{\prime}, \ldots, h_{k}^{\prime}\right) \in T\left(v_{1}, \ldots, v_{k}\right)$. Choose $\left(y_{1}, \ldots, y_{k}\right) \in S_{F_{1},\left(u_{1}, \ldots, u_{k}\right)} \times S_{F_{2},\left(u_{1}, \ldots, u_{k}\right)} \times \cdots \times S_{F_{k},\left(u_{1}, \ldots, u_{k}\right)}$ and $\left(y_{1}^{\prime}, \ldots, y_{k}^{\prime}\right) \in S_{F_{1},\left(v_{1}, \ldots, v_{k}\right)} \times S_{F_{2},\left(v_{1}, \ldots, v_{k}\right)} \times \cdots \times S_{F_{k},\left(v_{1}, \ldots, v_{k}\right)}$ such that

$$
h_{i}(t)=-\frac{(t-2) \frac{v_{i}-1}{\Gamma\left(v_{i}\right)\left(b+v_{i}-2\right) \frac{v_{i}-1}{h}}}{\sum_{s=2}^{b}}\left(b+v_{i}-\sigma(s)\right)^{\frac{v_{i}-1}{}} y_{i}(s)+\frac{1}{\Gamma\left(v_{i}\right)} \sum_{s=2}^{t-v_{i}}(t-\sigma(s))^{\frac{v_{i}-1}{}} y_{i}(s)
$$

and

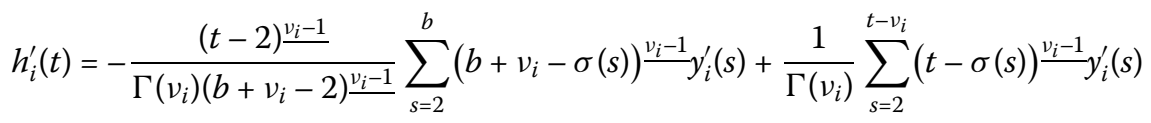

for all $t \in \mathbb{N}_{v_{i}-1}^{b+v_{i}}$ and $i=1, \ldots, k$. Since

$$
\begin{aligned}
H_{d} & \left(F _ { i } \left(t, u_{1}(t), \ldots, u_{k}(t), \Delta u_{1}(t), \ldots, \Delta u_{k}(t), \Delta^{2} u_{1}(t), \ldots, \Delta^{2} u_{k}(t),\right.\right. \\
& \left.\Delta_{v_{i}+1}^{\mu_{i 1}} u_{1}(t), \ldots, \Delta_{v_{i}+1}^{\mu_{i k}} u_{k}(t), \Delta_{v_{i}+1}^{\gamma_{i 1}} u_{1}(t), \ldots, \Delta_{v_{i}+1}^{\gamma_{i k}} u_{k}(t)\right), \\
& F_{i}\left(t, v_{1}(t), \ldots, v_{k}(t), \Delta v_{1}(t), \ldots, \Delta v_{k}(t), \Delta^{2} v_{1}(t), \ldots, \Delta^{2} v_{k}(t),\right. \\
& \left.\left.\Delta_{v_{i}+1}^{\mu_{i 1}} v_{1}(t), \ldots, \Delta_{v_{i}+1}^{\mu_{i k}} v_{k}(t), \Delta_{v_{i}+1}^{\gamma_{i 1}} v_{1}(t), \ldots, \Delta_{v_{i}+1}^{\gamma_{i k}} v_{k}(t)\right)\right) \\
\leq & \psi_{i}(t)\left(\sum _ { j = 1 } ^ { k } \left(\left|u_{j}(t)-v_{j}(t)\right|+\left|\Delta u_{j}(t)-\Delta v_{j}(t)\right|+\left|\Delta^{2} u_{j}(t)-\Delta^{2} v_{j}(t)\right|\right.\right. \\
& \left.\left.+\left|\Delta_{v_{i}+1}^{\mu_{i j}} u_{j}(t)-\Delta_{v_{i}+1}^{\mu_{i j}} v_{j}(t)\right|+\left|\Delta_{v_{i}+1}^{\gamma_{i j}} u_{j}(t)-\Delta_{v_{i}+1}^{\gamma_{i j}} v_{j}(t)\right|\right)\right)
\end{aligned}
$$

for all $\left(u_{1}, \ldots, u_{k}\right),\left(v_{1}, \ldots, v_{k}\right) \in \mathcal{X}$ and $t \in \mathbb{N}_{v_{i}-1}^{b+v_{i}}$, we get

$$
\begin{aligned}
\left|y_{i}(t)-y_{i}^{\prime}(t)\right| \leq & \psi_{i}(t)\left(\sum _ { j = 1 } ^ { k } \left(\left|u_{j}(t)-v_{j}(t)\right|+\left|\Delta u_{j}(t)-\Delta v_{j}(t)\right|+\left|\Delta^{2} u_{j}(t)-\Delta^{2} v_{j}(t)\right|\right.\right. \\
& \left.\left.+\left|\Delta_{v_{i}+1}^{\mu_{i j}} u_{j}(t)-\Delta_{v_{i}+1}^{\mu_{i j}} v_{j}(t)\right|+\left|\Delta_{v_{i}+1}^{\gamma_{i j}} u_{j}(t)-\Delta_{v_{i}+1}^{\gamma_{i j}} v_{j}(t)\right|\right)\right)
\end{aligned}
$$

for all $t \in \mathbb{N}_{2}^{b}$. Since

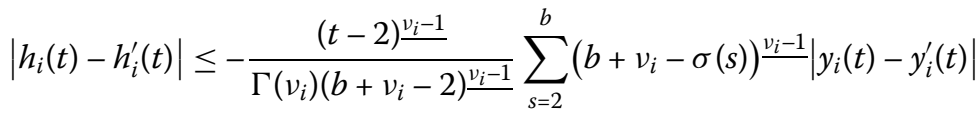

$$
\begin{aligned}
& +\frac{1}{\Gamma\left(v_{i}\right)} \sum_{s=2}^{t-v_{i}}(t-\sigma(s))^{\frac{v_{i}-1}{}}\left|y_{i}(t)-y_{i}^{\prime}(t)\right|
\end{aligned}
$$


and $\sum_{s=t-v_{i}+1}^{b} \frac{(t-\sigma(s))^{v_{i}-1}}{\Gamma(\nu)}=0$, we obtain

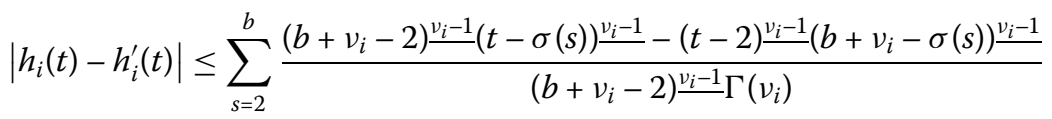

$$
\begin{aligned}
& \times\left|y_{i}(s)-y_{i}^{\prime}(s)\right| .
\end{aligned}
$$

Hence,

$$
\begin{aligned}
& \left|h_{i}(t)-h_{i}^{\prime}(t)\right| \leq \max _{t \in \mathbb{N}_{v_{i}-1}^{v_{i}+b}} \mid \frac{1}{\left(b+v_{i}-2\right) \frac{v_{i}-1}{\frac{v_{i}}{2}} \Gamma\left(v_{i}\right)} \sum_{s=2}^{b}\left(\left(b+v_{i}-2\right) \frac{v_{i}-1}{(t-\sigma(s))^{\frac{v_{i}-1}{}}}\right. \\
& \left.-(t-2) \stackrel{v_{i}-1}{\underline{v^{\prime}}}\left(b+v_{i}-\sigma(s)\right) \frac{v_{i}-1}{}\right) \mid \\
& \times \max _{t \in \mathbb{N}_{2}^{b}}\left|y_{i}(t)-y_{i}^{\prime}(t)\right| \\
& \leq \Lambda_{1}^{i} \max _{t \in \mathbb{N}_{2}^{b}}\left|y_{i}(t)-y_{i}^{\prime}(t)\right| \\
& \leq \Lambda_{1}^{i} \psi_{i}(t)\left(\sum _ { j = 1 } ^ { k } \left(\left|u_{j}(t)-v_{j}(t)\right|+\left|\Delta u_{j}(t)-\Delta v_{j}(t)\right|+\left|\Delta^{2} u_{j}(t)-\Delta^{2} v_{j}(t)\right|\right.\right. \\
& \left.\left.+\left|\Delta_{v_{i}+1}^{\mu_{i j}} u_{j}(t)-\Delta_{v_{i}+1}^{\mu_{i j}} v_{j}(t)\right|+\left|\Delta_{v_{i}+1}^{\gamma_{i j}} u_{j}(t)-\Delta_{v_{i}+1}^{\gamma_{i j}} v_{j}(t)\right|\right)\right) \\
& \leq \Lambda_{1}^{i}\left(\max _{t \in \mathbb{N}_{v_{i}-1}^{b+v_{i}}}\left|\psi_{i}(t)\right|\right)\left(\sum _ { j = 1 } ^ { k } \left(\max _{t \in \mathbb{N}_{v_{i}-1}^{b+v_{i}}}\left|u_{j}(t)-v_{j}(t)\right|+\max _{t \in \mathbb{N}_{v_{i}-1}^{b+v_{i}}}\left|\Delta u_{j}(t)-\Delta v_{j}(t)\right|\right.\right. \\
& +\max _{t \in \mathbb{N}_{v_{i}-1}^{b+v_{i}}}\left|\Delta^{2} u_{j}(t)-\Delta^{2} v_{j}(t)\right|+\max _{t \in \mathbb{N}_{v_{i}-1}^{b+v_{j}}}\left|\Delta_{v_{i}+1}^{\mu_{i j}} u_{j}(t)-\Delta_{v_{i}+1}^{\mu_{i j}} v_{j}(t)\right| \\
& \left.\left.+\max _{t \in \mathbb{N}_{v_{i}-1}^{+b v_{i}}}\left|\Delta_{v_{i}+1}^{\gamma_{i j}} u_{j}(t)-\Delta_{v_{i}+1}^{\gamma_{i j}} v_{j}(t)\right|\right)\right) \\
& \leq \Lambda_{1}^{i}\left(\max _{t \in \mathbb{N}_{v_{i}-1}^{b+v_{i}}}\left|\psi_{i}(t)\right|\right)\left(\sum_{i=1}^{k}\left\|u_{i}-v_{i}\right\|_{i}\right) \\
& \leq \Lambda_{1}^{i}\left(\max _{t \in \mathbb{N}_{v_{i}-1}^{b+v_{i}}}\left|\psi_{i}(t)\right|\right)\left\|\left(u_{1}-v_{1}, \ldots, u_{k}-v_{k}\right)\right\|_{\mathcal{X}}
\end{aligned}
$$

for all $t \in \mathbb{N}_{v_{i}-1}^{b+v_{i}}$. Since

$$
\begin{aligned}
\Delta h_{i}(t)= & -\frac{(t-2) \frac{v_{i}-2}{\Gamma\left(v_{i}-1\right)\left(b+v_{i}-2\right) \frac{v_{i}-1}{2}} \sum_{s=2}^{b}\left(b+v_{i}-\sigma(s)\right)^{\frac{v_{i}-1}{}} y_{i}(s)}{} \\
& +\frac{1}{\Gamma\left(v_{i}-1\right)} \sum_{s=2}^{t-v_{i}+1}(t-\sigma(s)) \frac{v_{i}-2}{y_{i}}(s),
\end{aligned}
$$


we get

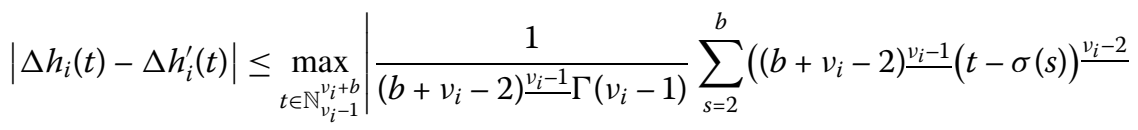

$$
\begin{aligned}
& -(t-2) \frac{v_{i}-2}{\left.\left(b+v_{i}-\sigma(s)\right) \frac{v_{i}-1}{}\right)} \mid \\
& \times \max _{t \in \mathbb{N}_{0}^{b}}\left|y_{i}(t)-y_{i}^{\prime}(t)\right| \\
& \leq \Lambda_{2}^{i} \max _{t \in \mathbb{N}_{0}^{b}}\left|y_{i}(t)-y_{i}^{\prime}(t)\right| \\
& \vdots \\
& \leq \Lambda_{2}^{i}\left(\max _{t \in \mathbb{N}_{v_{i}-1}^{v_{i}+b}}\left|\psi_{i}(t)\right|\right)\left\|\left(u_{1}-v_{1}, \ldots, u_{k}-v_{k}\right)\right\|_{\mathcal{X}}
\end{aligned}
$$

for all $t \in \mathbb{N}_{v_{i}-1}^{b+v_{i}}$. Since

$$
\begin{aligned}
\Delta^{2} h_{i}(t)= & -\frac{(t-2) \frac{v_{i}-3}{\Gamma\left(v_{i}-2\right)\left(b+v_{i}-2\right) \frac{v_{i}-1}{}} \sum_{s=2}^{b}\left(b+v_{i}-\sigma(s)\right) \frac{v_{i}-1}{y_{i}} y_{i}(s)}{}+\frac{1}{\Gamma\left(v_{i}-2\right)} \sum_{s=2}^{t-v_{i}+2}(t-\sigma(s)) \frac{v_{i}-3}{n} y_{i}(s),
\end{aligned}
$$

we get

$$
\begin{aligned}
& \left|\Delta^{2} h_{i}(t)-\Delta^{2} h_{i}^{\prime}(t)\right| \leq \max _{t \in \mathbb{N}_{v_{i}-1}^{v_{i}+b}} \mid \frac{1}{\left(b+v_{i}-2\right) \frac{v_{i}-1}{\Gamma} \Gamma\left(v_{i}-2\right)} \sum_{s=2}^{b}\left(\left(b+v_{i}-2\right) \frac{v_{i}-1}{(t-\sigma(s)) \frac{v_{i}-3}{}}\right. \\
& -(t-2) \frac{v_{i}-3}{\left.\left(b+v_{i}-\sigma(s)\right) \frac{v_{i}-1}{}\right)} \\
& \times \max _{t \in \mathbb{N}_{0}^{b}}\left|y_{i}(t)-y_{i}^{\prime}(t)\right| \\
& \leq \Lambda_{3}^{i} \max _{t \in \mathbb{N}_{0}^{b}}\left|y_{i}(t)-y_{i}^{\prime}(t)\right| \\
& \leq \Lambda_{3}^{i}\left(\max _{t \in \mathbb{N}_{v_{i}-1}^{v_{i}+b}}\left|\psi_{i}(t)\right|\right)\left\|\left(u_{1}-v_{1}, \ldots, u_{k}-v_{k}\right)\right\|_{\mathcal{X}}
\end{aligned}
$$

for all $t \in \mathbb{N}_{v_{i}-1}^{b+v_{i}}$. Using Lemma 1.1, we obtain

$$
\begin{aligned}
\Delta_{v_{i}+1}^{\mu_{i i}} h_{1}(t)= & \frac{-(t-2) \frac{v_{i}-\mu_{i i}-1}{\Gamma\left(v_{i}-\mu_{i i}\right)\left(b+v_{i}-2\right) \frac{v_{i}-1}{}} \sum_{s=0}^{b}\left(v_{i}+b-\sigma(s)\right) \frac{v_{i}-1}{y_{i}} y_{i}(s)}{} \\
& +\sum_{s=2}^{t-v_{i}+\mu_{i i}} \frac{(t-\sigma(s)) \frac{v_{i}-1-\mu_{i i}}{\Gamma\left(v_{i}-\mu_{i i}\right)}}{y_{i}}(s),
\end{aligned}
$$


and so

$$
\begin{aligned}
& \left|\Delta_{v_{i}+1}^{\mu_{i i}} h_{i}(t)-\Delta_{v_{i}+1}^{\mu_{i i}} h_{i}^{\prime}(t)\right|
\end{aligned}
$$

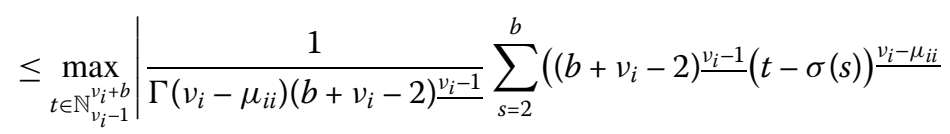

$$
\begin{aligned}
& \left.-(t-2) \frac{v_{i}-\mu_{i i}}{\underline{n_{i}}}\left(b+v_{i}-\sigma(s)\right)^{\frac{v_{i}-1}{}}\right) \\
& \times \max _{t \in \mathbb{N}_{0}^{b}}\left|y_{i}(t)-y_{i}^{\prime}(t)\right| \\
& \leq \Lambda_{4}^{i} \max _{t \in \mathbb{N}_{0}^{b}}\left|y_{i}(t)-y_{i}^{\prime}(t)\right| \\
& \vdots \\
& \leq \Lambda_{4}^{i}\left(\max _{t \in \mathbb{N}_{v_{i} v^{i}+b}}\left|\psi_{i}(t)\right|\right)\left\|\left(u_{1}-v_{1}, \ldots, u_{k}-v_{k}\right)\right\|_{\mathcal{X}}
\end{aligned}
$$

for $t \in \mathbb{N}_{v_{i}-1}^{b+v_{i}}$, and similarly

$$
\left|\Delta_{v_{i}+1}^{\gamma_{i i}} h_{i}(t)-\Delta_{v_{i}+1}^{\gamma_{i i}} h_{i}^{\prime}(t)\right| \leq \Lambda_{5}^{i}\left(\max _{t \in \mathbb{N}_{v_{i}-1}^{b+v_{i}}}^{i}\left|\psi_{i}(t)\right|\right)\left\|\left(u_{1}-v_{1}, \ldots, u_{k}-v_{k}\right)\right\|_{\mathcal{X}}
$$

for $t \in \mathbb{N}_{v_{i}-1}^{b+v_{i}}$. Thus,

$$
\begin{aligned}
\left\|h_{i}-h_{i}^{\prime}\right\|_{i}= & \max _{t \in \mathbb{N}_{v_{i}-1}^{b+v_{i}}}\left|h_{i}(t)-h_{i}^{\prime}(t)\right|+\max _{t \in \mathbb{N}_{v_{i}-1}^{b+v_{i}}}\left|\Delta h_{i}(t)-\Delta h_{i}^{\prime}(t)\right|+\max _{t \in \mathbb{N}_{v_{i}-1}^{b+v_{i}}}\left|\Delta^{2} h_{i}(t)-\Delta^{2} h_{i}^{\prime}(t)\right| \\
& +\max _{t \in \mathbb{N}_{v_{i}-1}^{b+v_{i}}}\left|\Delta_{v_{i}+1}^{\mu_{i i}} h_{i}(t)-\Delta_{v_{i}+1}^{\mu_{i i}} h_{i}^{\prime}(t)\right|+\max _{t \in \mathbb{N}_{v_{i}-1}^{b+v_{i}}}\left|\Delta_{v_{i}+1}^{\gamma_{i i}} h_{i}(t)-\Delta_{v_{i}+1}^{\gamma_{i i}} h_{i}^{\prime}(t)\right| \\
\leq & \left(\Lambda_{1}^{i}+\cdots+\Lambda_{5}^{i}\right)\left(\max _{t \in \mathbb{N}_{v_{i}-1}^{b+v_{i}}}\left|\psi_{i}(t)\right|\right)\left\|\left(u_{1}-v_{1}, \ldots, u_{k}-v_{k}\right)\right\|_{\mathcal{X}}
\end{aligned}
$$

for $i=1, \ldots, k$ and all $\left(u_{1}, \ldots, u_{k}\right),\left(v_{1}, \ldots, v_{k}\right) \in \mathcal{X}, h_{i} \in T\left(u_{1}, \ldots, u_{k}\right)$, and $h_{i}^{\prime} \in T\left(v_{1}, \ldots, v_{k}\right)$. This implies that

$$
\begin{aligned}
& \left\|\left(h_{1}, \ldots, h_{k}\right)-\left(h_{1}^{\prime}, \ldots, h_{k}^{\prime}\right)\right\|_{\mathcal{X}} \\
& \quad=\sum_{i=1}^{k}\left\|h_{i}-h_{i}^{\prime}\right\|_{i} \\
& \leq \sum_{i=1}^{k}\left(\left(\Lambda_{1}^{i}+\cdots+\Lambda_{5}^{i}\right)\left(\max _{t \in \mathbb{N}_{v_{i}-1}^{b+v_{i}}}\left|\psi_{i}(t)\right|\right)\left\|\left(u_{1}-v_{1}, \ldots, u_{k}-v_{k}\right)\right\|_{\mathcal{X}}\right) \\
& \leq\left(\sum_{i=1}^{k}\left(\max _{t \in \mathbb{N}_{v_{i}-1}^{b+v_{i}}}\left|\psi_{i}(t)\right|\right)\left(\Lambda_{1}^{i}+\cdots+\Lambda_{5}^{i}\right)\right)\left\|\left(u_{1}-v_{1}, \ldots, u_{k}-v_{k}\right)\right\| \\
& \quad=L\left\|\left(u_{1}-v_{1}, \ldots, u_{k}-v_{k}\right)\right\| .
\end{aligned}
$$


By the result of Covitz and Nadler there exists $x^{*} \in \mathcal{X}$ such that $x^{*} \in T\left(x^{*}\right)$. We can check that $x^{*}$ is a solution for the system of fractional difference inclusions (1).

The following example illustrates our main result.

Example 1 Consider the two-dimensional system of fractional difference inclusions

$$
\left\{\begin{aligned}
\Delta_{1.7}^{2.7} x_{1}(t) \in & {\left[0,2 \pi+e^{4 t}+\frac{\sin x_{1}(t)}{e^{t+4}}+\frac{\sin x_{2}(t)}{3 e^{t+3}}+\frac{\left|\Delta x_{1}(t)\right|+\left|\Delta x_{2}(t)\right|}{e^{10}}\right.} \\
& +\frac{\cos \sin \left(\Delta^{2} x_{1}(t)\right)}{3 e^{t+3}}+\frac{\cos \sin \left(\Delta^{2} x_{2}(t)\right)}{e^{t+4}}+\frac{\left|\Delta_{3.7}^{0.6} x_{1}(t)\right|}{\cosh (t+10)}+\frac{\left|\Delta_{3.7}^{0.5} x_{2}(t)\right|}{t e^{t+2}}+\frac{\left|\Delta_{3.7}^{1.6} x_{1}(t)\right|}{\pi e^{t+3}} \\
& \left.+\frac{\left|\Delta_{3.7}^{1.2} x_{2}(t)\right|}{e^{t+4}}\right] \\
\Delta_{1.3}^{2.3} x_{2}(t) \in & {\left[0,4+e^{t^{2}}+\frac{\left|x_{1}(t)\right|}{e^{t^{2}+4}}+\frac{\left|x_{2}(t)\right|}{e^{t^{2}+3}}+\frac{\left|\Delta x_{1}(t)\right|}{e^{t t^{2}}}+\frac{\left|\Delta x_{2}(t)\right|}{\cosh \left(t^{5}+8\right)}\right.} \\
& \left.+\frac{\sin \left(\Delta^{2} x_{1}(t)\right)}{3 e^{t^{2}+3}}+\frac{\sin \left(\Delta^{2} x_{1}(t)\right)}{e^{t^{4}+4}}+\frac{\left|\Delta_{3.3}^{0.8} x_{1}(t)\right|+\left|\Delta_{3.3}^{0.4} x_{2}(t)\right|}{e^{t^{4}+6}}+\frac{\left|\Delta_{3.3}^{1.9} x_{1}(t)\right|}{4 e^{t^{2}+1}}+\frac{\left|\Delta_{3.3}^{1.3} x_{2}(t)\right|}{t^{2} e^{t^{4}}}\right]
\end{aligned}\right.
$$

with the boundary conditions $x_{1}(1.7)=x_{1}(2.7)=x_{1}(7.7)=0$ and $x_{1}(1.3)=x_{1}(2.3)=x_{1}(7.3)=$ 0 . Put $b=5, v_{1}=2.7, \mu_{11}=0.6, \mu_{12}=0.5, \gamma_{11}=1.6, \gamma_{12}=1.2, v_{2}=2.3, \mu_{21}=0.8, \mu_{22}=0.4$, $\gamma_{21}=1.9, \gamma_{22}=1.3$,

$$
\begin{aligned}
F_{1}\left(t, y_{1}, \ldots, y_{10}\right)= & {\left[0,2 \pi+e^{4 t}+\frac{\sin y_{1}}{e^{t+4}}+\frac{\sin y_{2}}{3 e^{t+3}}+\frac{\left|y_{3}\right|+\left|y_{4}\right|}{e^{10}}\right.} \\
& \left.+\frac{\cos \sin y_{5}}{3 e^{t+3}}+\frac{\cos \sin y_{6}}{e^{t+4}}+\frac{\left|y_{7}\right|}{\cosh (t+10)}+\frac{\left|y_{8}\right|}{t e^{t+2}}+\frac{\left|y_{9}\right|}{\pi e^{t+3}}+\frac{\left|y_{10}\right|}{e^{t+4}}\right],
\end{aligned}
$$

and

$$
\begin{aligned}
F_{2}\left(t, y_{1}, \ldots, y_{10}\right)= & {\left[0,4+e^{t^{2}}+\frac{\left|y_{1}\right|}{e^{t^{2}+4}}+\frac{\left|y_{2}\right|}{e^{t^{2}+3}}+\frac{\left|y_{3}\right|}{e^{\pi t^{2}}}+\frac{\left|y_{4}\right|}{\cosh \left(t^{5}+8\right)}\right.} \\
& \left.+\frac{\sin y_{5}}{3 e^{t^{2}+3}}+\frac{\sin y_{6}}{e^{t^{4}+4}}+\frac{\left|y_{7}\right|+\left|y_{8}\right|}{e^{t^{4}+6}}+\frac{\left|y_{9}\right|}{4 e^{t^{2}+1}}+\frac{\left|y_{10}\right|}{t^{2} e^{t^{4}}}\right] .
\end{aligned}
$$

Note that $2 \pi+e^{4 t}+\frac{\sin y_{1}}{e^{t+4}}+\frac{\sin y_{2}}{3 e^{t+3}}+\frac{\left|y_{3}\right|+\left|y_{4}\right|}{e^{10}}+\frac{\cos \sin y_{5}}{3 e^{t+3}}+\frac{\cos \sin y_{6}}{e^{t+4}}+\frac{\left|y_{7}\right|}{\cosh (t+10)}+\frac{\left|y_{8}\right|}{t e^{t+2}}+\frac{\left|y_{9}\right|}{\pi e^{t+3}}+\frac{\left|y_{10}\right|}{e^{t+4}}>0$ for $t \in \mathbb{N}_{1.7}^{7.7}$ and $y_{1}, \ldots, y_{10} \in \mathbb{R}$, and so $F_{1}: \mathbb{N}_{1.7}^{7.7} \times \mathbb{R}^{10} \rightarrow 2^{\mathbb{R}}$ is a nonempty-valued multifunction. If $\psi_{1}(t)=\frac{1}{e^{(t+2)}}$, then $\max _{t \in \mathbb{N}_{1.7}^{7.7}}\left|\psi_{1}(t)\right|=\max _{t \in \mathbb{N}_{1.7}^{7.7}} \frac{1}{e^{t+2}}=\frac{1}{e^{3.7}} \cong \frac{1}{40.4473}$. Similarly, we have $4+e^{t^{2}}+\frac{\left|y_{1}\right|}{e^{t^{2}+4}}+\frac{\left|y_{2}\right|}{e^{t^{2}+3}}+\frac{\left|y_{3}\right|}{e^{\pi t^{2}}}+\frac{\left|y_{4}\right|}{\cosh \left(t^{5}+8\right)}+\frac{\sin y_{5}}{3 e^{t^{2}+3}}+\frac{\sin y_{6}}{e^{t^{4}+4}}+\frac{\left|y_{7}\right|+\left|y_{8}\right|}{e^{t^{4}+6}}+\frac{\left|y_{9}\right|}{4 e^{t^{2}+1}}+\frac{\left|y_{10}\right|}{t^{2} e^{t^{4}}}>0$ for $t \in \mathbb{N}_{1.3}^{7.3}$ and $y_{1}, \ldots, y_{10} \in \mathbb{R}$, and so $F_{2}: \mathbb{N}_{1.3}^{7.3} \times \mathbb{R}^{10} \rightarrow 2^{\mathbb{R}}$ is a nonempty-valued multifunction. If $\psi_{2}(t)=\frac{1}{e^{\left(t^{2}+2\right)}}$, then $\max _{t \in \mathbb{N}_{1.3}^{7.3}}\left|\psi_{2}(t)\right|=\max _{t \in \mathbb{N}_{1.3}^{7.3}} \frac{1}{e^{\left(t^{2}+2\right)}}=\frac{1}{e^{\left((1.3)^{2}+2\right)}} \cong \frac{1}{40.0448}$. Note that

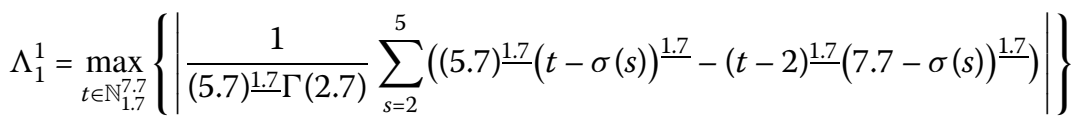

$$
\begin{aligned}
& \cong \max \{0.0,0.0,1.48,3,3.7,2.89,0\}=3.7 \text {, }
\end{aligned}
$$

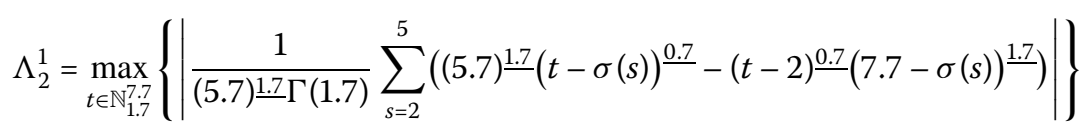

$$
\begin{aligned}
& \cong \max \{0,1.48,1.51,0.7,0.8,2.89,4.53\}=4.53 \text {, }
\end{aligned}
$$




$$
\begin{aligned}
& \Lambda_{3}^{1}=\max _{t \in \mathbb{N}_{1.7}^{7.7}}\left\{\left|\frac{1}{(5.7) \frac{1.7}{\Gamma} \Gamma(0.7)} \sum_{s=2}^{5}\left((5.7)^{\frac{1.7}{.}}(t-\sigma(s))^{\frac{-0.3}{}}-(t-2)^{-\frac{-0.3}{(}}(7.7-\sigma(s))^{\frac{1.7}{1.7}}\right)\right|\right\} \\
& \cong \max \{1.48,0.03,0.81,1.5,2.09,1.63,1.43\}=2.09 \text {, } \\
& \Lambda_{4}^{1}=\max _{t \in \mathbb{N}_{1.7}^{7.7}}\left\{\left|\frac{1}{\Gamma(2.1)(5.7) \frac{1.7}{2}} \sum_{s=2}^{5}\left((5.7)^{1.7}(t-\sigma(s))^{\frac{2.1}{1}}-(t-2)^{2.1}(7.7-\sigma(s))^{1.7}\right)\right|\right\} \\
& \cong \max \{1.87,1.11,0.88,0.77,0.59,0.25,0.16\}=1.87 \text {, } \\
& \Lambda_{5}^{1}=\max _{t \in \mathbb{N}_{1.7}^{7.7}}\left\{\left|\frac{1}{\Gamma(1.1)(5.7) \underline{1.7}} \sum_{s=2}^{5}\left((5.7)^{1.7}(t-\sigma(s))^{\frac{1.1}{1}}-(t-2)^{\underline{1.1}}(7.7-\sigma(s))^{\frac{1.7}{}}\right)\right|\right\} \\
& \cong \max \{1.35,0.39,0.2,2.42,0.59,0.17,0.08\}=2.42 \text {, } \\
& \left.\Lambda_{1}^{2}=\max _{t \in \mathbb{N}_{1.3}^{7.3}}\left|\frac{1}{(5.3) \underline{1.3} \Gamma(2.3)} \sum_{s=2}^{5}\left((5.3)^{\frac{1.3}{2}}(t-\sigma(s))^{1.3}-(t-2)^{\frac{1.3}{3}}(7.3-\sigma(s))^{1.3}\right)\right|\right\} \\
& \cong \max \{0,0,1.73,3,3.3,2.36,0\}=3.3 \text {, } \\
& \Lambda_{2}^{2}=\max _{t \in \mathbb{N}_{1.3}^{7.3}}\left\{\left|\frac{1}{(5.3) \underline{1.3} \Gamma(1.3)} \sum_{s=2}^{5}\left((5.3) \frac{1.3}{-}(t-\sigma(s))^{\frac{0.3}{n}}-(t-2)^{0.3}(7.3-\sigma(s))^{1.3}\right)\right|\right\} \\
& \cong \max \{0,1.73,1.26,0.3,0.93,2.36,2.94\}=2.94 \text {, } \\
& \Lambda_{3}^{2}=\max _{t \in \mathbb{N}_{1.3}^{7.3}}\left\{\left|\frac{1}{(5.3) \frac{1.3}{.3} \Gamma(0.3)} \sum_{s=2}^{5}\left((5.3)^{1.3}(t-\sigma(s))^{\frac{-0.7}{2}}-(t-2)^{\frac{-0.7}{2}}(7.3-\sigma(s))^{\frac{1.3}{3}}\right)\right|\right\} \\
& \cong \max \{1.73,0.47,0.96,1.23,1.43,0.58,0.41\}=1.73 \text {, } \\
& \Lambda_{4}^{2}=\max _{t \in \mathbb{N}_{1.3}^{7.3}}\left\{\left|\frac{1}{\Gamma(1.9)(5.3) \underline{1.3}} \sum_{s=2}^{5}\left((5.3)^{1.3}(t-\sigma(s))^{\frac{1.9}{1.9}}-(t-2)^{1.9}(7.3-\sigma(s))^{1.3}\right)\right|\right\} \\
& \cong \max \{3.76,0.82,0.46,0.33,1.43,0.29,0.14\}=3.76 \text {, }
\end{aligned}
$$

and

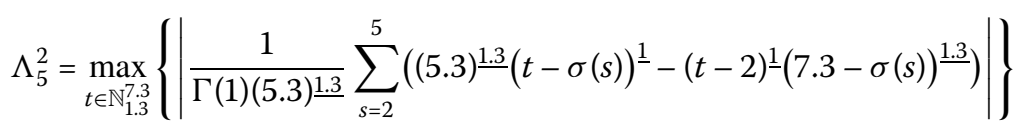

$$
\begin{aligned}
& \cong \max \{4.68,0.56,0.21,2.81,1.82,0.23,0.088\}=4.68 \text {. }
\end{aligned}
$$

Thus, we obtain the table:

\begin{tabular}{llllll}
\hline & $\boldsymbol{\Lambda}_{\boldsymbol{j}}^{\boldsymbol{i}}$ & & & & \\
\cline { 2 - 6 } & $\boldsymbol{j = \mathbf { 1 }}$ & $\boldsymbol{j = 2}$ & $\boldsymbol{j = 3}$ & $\boldsymbol{j = 4}$ & $\boldsymbol{j = 5}$ \\
\hline$i=1$ & 3.7 & 4.53 & 2.09 & 1.87 & 2.42 \\
$i=2$ & 3.3 & 2.94 & 1.73 & 3.76 & 4.68 \\
\hline
\end{tabular}

Note that

$$
0<L=\sum_{i=1}^{2}\left(\max _{t \in \mathbb{N}_{v_{i}-1}^{5+v_{i}}}\left|\psi_{i}(t)\right|\right)\left(\Lambda_{1}^{i}+\cdots+\Lambda_{5}^{i}\right)
$$




$$
\begin{aligned}
& \cong \frac{1}{40.44}(3.7+4.53+2.09+1.87+2.42)+\frac{1}{40.04}(3.3+2.94+1.73+3.76+4.68) \\
& =\frac{1}{40.44}(14.63)+\frac{1}{40.04}(16.44) \cong 0.77<1 .
\end{aligned}
$$

On the other hand, we have

$$
\begin{aligned}
H_{d} & \left.F_{1}\left(t, y_{1}, \ldots, y_{10}\right), F_{1}\left(t, z_{1}, \ldots, z_{10}\right)\right) \\
\leq & \mid \frac{\sin y_{1}}{e^{t+4}}+\frac{\sin y_{2}}{3 e^{t+3}}+\frac{\left|y_{3}\right|+\left|y_{4}\right|}{e^{10}}+\frac{\cos \sin y_{5}}{3 e^{t+3}} \\
& +\frac{\cos \sin y_{6}}{e^{t+4}}+\frac{\left|y_{7}\right|}{\cosh (t+10)}+\frac{\left|y_{8}\right|}{t e^{t+2}}+\frac{\left|y_{9}\right|}{\pi e^{t+3}}+\frac{\left|y_{10}\right|}{e^{t+4}} \\
& \quad-\frac{\sin z_{1}}{e^{t+4}}-\frac{\sin z_{2}}{3 e^{t+3}}-\frac{\left|z_{3}\right|+\left|z_{4}\right|}{e^{10}}-\frac{\cos \sin z_{5}}{3 e^{t+3}} \\
& \quad-\frac{\cos \sin z_{6}}{e^{t+4}}-\frac{\left|z_{7}\right|}{\cosh (t+10)}-\frac{\left|z_{8}\right|}{t e^{t+2}}-\frac{\left|z_{9}\right|}{\pi e^{t+3}}-\frac{\left|z_{10}\right|}{e^{t+4}} \mid \\
\leq & \frac{1}{e^{(t+2)}}\left(\sin y_{1}-\sin z_{1}+\sin y_{2}-\sin z_{2}+\left|y_{3}-z_{3}\right|+\left|y_{4}-z_{4}\right|+\cos \sin y_{5}-\cos \sin z_{5}\right. \\
& \left.+\cos \sin y_{6}-\cos \sin z_{6}+\left|y_{7}-z_{7}\right|+\left|y_{8}-z_{8}\right|+\left|y_{9}-z_{9}\right|+\left|y_{10}-z_{10}\right|\right) \\
\leq & \frac{1}{e^{(t+2)}}\left(\sum_{i=1}^{10}\left|y_{i}-z_{i}\right|\right) \leq \psi_{1}(t) \sum_{k=1}^{10}\left|y_{k}-z_{k}\right|
\end{aligned}
$$

for all $t \in \mathbb{N}_{1.7}^{7.7}, y_{1}, \ldots, y_{10}, z_{1}, \ldots, z_{10} \in \mathbb{R}$. Similarly, we have

$$
\begin{aligned}
H_{d}( & \left.F_{2}\left(t, y_{1}, \ldots, y_{10}\right), F_{2}\left(t, z_{1}, \ldots, z_{10}\right)\right) \\
\leq & \mid \frac{\left|y_{1}\right|}{e^{t^{2}+4}}+\frac{\left|y_{2}\right|}{e^{t^{2}+3}}+\frac{\left|y_{3}\right|}{e^{\pi t^{2}}}+\frac{\left|y_{4}\right|}{\cosh \left(t^{5}+8\right)} \\
& +\frac{\sin y_{5}}{3 e^{t^{2}+3}}+\frac{\sin y_{6}}{e^{t^{4}+4}}+\frac{\left|y_{7}\right|+\left|y_{8}\right|}{e^{t^{4}+6}}+\frac{\left|y_{9}\right|}{4 e^{t^{2}+1}}+\frac{\left|y_{10}\right|}{t^{2} e^{t^{4}}} \\
& -\frac{\left|z_{1}\right|}{e^{t^{2}+4}}-\frac{\left|z_{2}\right|}{e^{t^{2}+3}}-\frac{\left|z_{3}\right|}{e^{\pi t^{2}}}-\frac{\left|z_{4}\right|}{\cosh \left(t^{5}+8\right)} \\
& -\frac{\sin z_{5}}{3 e^{t^{2}+3}}-\frac{\sin z_{6}}{e^{t^{4}+4}}-\frac{\left|z_{7}\right|+\left|z_{8}\right|}{e^{t^{4}+6}}-\frac{\left|z_{9}\right|}{4 e^{t^{2}+1}}-\frac{\left|z_{10}\right|}{t^{2} e^{t^{4}}} \mid \\
\leq & \frac{1}{e^{\left(t^{2}+2\right)}}\left(\left|y_{1}-z_{1}\right|+\left|y_{2}-z_{2}\right|+\left|y_{3}-z_{3}\right|+\left|y_{4}-z_{4}\right|+\sin y_{5}-\sin z_{5}\right. \\
& \left.+\sin y_{6}-\sin z_{6}+\left|y_{7}-z_{7}\right|+\left|y_{8}-z_{8}\right|+\left|y_{9}-z_{9}\right|+\left|y_{10}-z_{10}\right|\right) \\
\leq & \frac{1}{e^{\left(t^{2}+2\right)}}\left(\sum_{i=1}^{10}\left|y_{i}-z_{i}\right|\right) \leq \psi_{2}(t) \sum_{k=1}^{10}\left|y_{k}-z_{k}\right|
\end{aligned}
$$

for all $t \in \mathbb{N}_{1.7}^{7.7}, y_{1}, \ldots, y_{10}, z_{1}, \ldots, z_{10} \in \mathbb{R}$. Now, using Theorem 2.2, we conclude that problem (3) has a solution. 


\section{Competing interests}

The authors declare that they have no competing interests.

\section{Authors' contributions}

Both authors have equal contributions. The whole work was carried out, read, and approved by the authors.

\section{Publisher's Note}

Springer Nature remains neutral with regard to jurisdictional claims in published maps and institutional affiliations.

Received: 19 June 2017 Accepted: 11 September 2017 Published online: 13 October 2017

\section{References}

1. Almeida, R, Malinowska, AB, Odzijewicz, T: Fractional differential equations with dependence on the Caputo-Katugampola derivative. J. Comput. Nonlinear Dyn. 11(6), 061017 (2016). doi:10.1115/1.4034432

2. Alsaedi, A, Baleanu, D, Etemad, S, Rezapour, Sh: On coupled systems of time-fractional differential problems by using a new fractional derivative. J. Funct. Spaces 2016, Article ID 4626940 (2016)

3. Gao, F, Yang, XJ: Fractional Maxwell fluid with fractional derivative without singular kernel. Therm. Sci. 20(3), 871-877 (2016)

4. Yang, XJ: Fractional derivatives of constant and variable orders applied to anomalous relaxation models in heat-transfer problems. Therm. Sci. 21(3), 1161-1171 (2017)

5. Yang, XJ, Machado, JAT: A new fractional operator of variable order: application in the description of anomalous diffusion. Physica A 481, 276-283 (2017)

6. Yang, XJ, Srivastava, HM, Machado, JAT: A new fractional derivative without singular kernel: application to the modelling of the steady heat flow. Therm. Sci. 20(2), 753-756 (2016)

7. Wu, GC, Baleanu, D, Deng, ZG, Zeng, SD: Lattice fractional diffusion equation in terms of a Riesz-Caputo difference. Physica A 438, 335-339 (2015)

8. Wu, GC, Baleanu, D, Xie, HP: Riesz Riemann-Liouville difference on discrete domains. Chaos 26, Article ID 084308 (2016)

9. Acar, N, Atici, FM: Exponential functions of discrete fractional calculus. Appl. Anal. Discrete Math. 7, $343-353$ (2013)

10. Atici, FM, Eloe, PW: Initial value problems in discrete fractional calculus. Proc. Am. Math. Soc. 137, $981-989$ (2009)

11. Holm, M: Sum and differences compositions in discrete fractional calculus. CUBO 13, 153-184 (2011)

12. Holm, M: The theory of discrete fractional calculus: development and applications. Ph.D. thesis, University of Nebraska-Lincoln, Ann Arbor, MI (2011)

13. Jarad, F, Kaymakcalan, B, Tas, K: A new transform method in nabla discrete fractional calculus. Adv. Differ. Equ. 2012, $190(2012)$

14. Agarwal, RP, Baleanu, D, Rezapour, Sh, Salehi, S: The existence of solutions for some fractional finite difference equations via sum boundary conditions. Adv. Differ. Equ. 2014, 282 (2014)

15. Atici, FM, Sengul, S: Modeling with fractional difference equations. J. Math. Anal. Appl. 369, 1-9 (2010)

16. Baleanu, D, Rezapour, Sh, Salehi, S: A k-dimensional system of fractional finite difference equations. Abstr. Appl. Anal. 2014, Article ID 312578 (2014)

17. Baleanu, D, Rezapour, Sh, Salehi, S: On some self-adjoint fractional finite difference equations. J. Comput. Anal. Appl. $19,59-67(2015)$

18. Baleanu, D, Rezapour, Sh, Salehi, S: On the existence of solutions for a fractional finite difference inclusion via three points boundary conditions. Adv. Differ. Equ. 2015, 242 (2015)

19. Baleanu, D, Rezapour, Sh, Salehi, S: A fractional finite difference inclusion. J. Comput. Anal. Appl. 20(5), 834-842 (2016)

20. Dassios, IK, Baleanu, D: On a singular system of fractional nabla difference equations with boundary conditions. Bound. Value Probl. 2013, 148 (2013)

21. Goodrich, ChS: On a fractional boundary value problem with fractional boundary conditions. Appl. Math. Lett. 25 1101-1105 (2012)

22. Goodrich, ChS: On discrete sequential fractional boundary value problems. J. Math. Anal. Appl. 385, 111-124 (2012)

23. Kang, S, Li, Y, Chen, H: Positive solutions to boundary value problems of fractional difference equation with nonlocal conditions. Adv. Differ. Equ. 2014, 7 (2014)

24. Mohan, JJ, Deekshitulu, GVSR: Fractional order difference equations. Int. J. Differ. Equ. 2012, 780619 (2012)

25. Rezapour, Sh, Salehi, S: On the existence of solution for a $k$-dimensional system of three points nabla fractional finite difference equations. Bull. Iran. Math. Soc. 41(6), 1433-1444 (2015)

26. Weidong, L, Feng, J: Nonlinear discrete fractional mixed type sum-difference equation boundary value problems in Banach spaces. Adv. Differ. Equ. 2014, 184 (2014)

27. Anastassiou, GA: Nabla discrete fractional calculus and nabla inequalities. Math. Comput. Model. 51, 562-571 (2010)

28. Atici, FM, Eloe, PW: Discrete fractional calculus with the nabla operator. Electron. J. Qual. Theory Differ. Equ. 2009, 3 2009)

29. Dassios, LK, Baleanu, D, Kalogeropoulos, Gl: On non-homogeneous singular systems of fractional nabla difference equations. Appl. Math. Comput. 227, 112-131 (2014)

30. Jia, B, Erbe, L, Peterson, A: Two monotonicity results for nabla and delta fractional differences. Arch. Math. 104(6), 589-597 (2015)

31. Yang, XJ, Baleanu, D, Srivastava, HM: Local Fractional Integral Transforms and Their Applications. Academic Press, San Diego (2015)

32. Awasthi, P: Boundary value problems for discrete fractional equations. Ph.D. thesis, University of Nebraska-Lincoln, Ann Arbor, MI (2013)

33. Aleomraninejad, SMA, Rezapour, Sh, Shahzad, N: On generalizations of the Suzuki's method. Appl. Math. Lett. 24, 1037-1040 (2011)

34. Berinde, V, Pacurar, M: The role of the Pompeiu-Hausdorff metric in fixed point theory. Creative Math. Inform. 22(2), 35-42 (2013)

35. Covitz, H, Nadler, S: Multivalued contraction mappings in generalized metric spaces. Isr. J. Math. 8, 5-11 (1970)

36. Goodrich, ChS: A comparison result for the fractional difference operator. Int. J. Differ. Equ. 6, 17-37 (2011) 